\title{
Exploring the Patient's Experience of Receiving Clinical Care Which Incorporates the Use of Mobile Technology at the Bedside
}

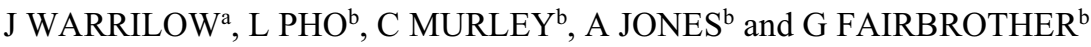 \\ ${ }^{a}$ The Canterbury Hospital, Campsie New South Wales, Australia \\ ${ }^{\mathrm{b}}$ Sydney Local Health District, Camperdown, New South Wales, Australia
}

\begin{abstract}
Objective: Research-based insight into patient's experiences of mobile technology at the bedside in the hospital setting remains limited. This research project aims to explore patient's experience. Methods: This mixed method pre and post study aimed to explore the patient experience in relation to this and also test whether introducing further bedside technology (beyond the workstation on wheels) had an effect on the patient experience. Questionnaires and interviews were conducted among inpatient samples prior to and one year post introduction of a suite of new bedside technologies. Results: Pre and post patient survey results (pre: $\mathrm{n}=82$; post: $\mathrm{n}=98$ ) suggested that mixed views and perceptions existed and that some of these were associated with primary demographics such as age. At post-test, attitudes about bedside technology were found to be more positive, and feedback about care quality was found to be unchanged, Baseline patient interview findings $(n=15)$ highlight the social ubiquity of technology as a driver of positive attitude in the digital health context. Conclusion: The addition of new bedside technology is very well received by patients and was not perceived to impact on care quality.
\end{abstract}

Keywords. Patient experience, digital health technology, patient care, consumer experience

\section{Introduction}

It is widely accepted that positive patient experience is linked with improved health outcomes and engagement with health services [1][2]. In recent years there has been a proliferation of bedside technology in our inpatient healthcare environments. However there has been little research on the patient experience and perception of bedside information technology such as computers on wheels and similar hardware.

Electronic medical records (eMRs) are a digital version of the paper charts clinicians previously used within a health care setting. eMRs contain the medical and treatment histories of patients in one central virtual location and allow clinical data to be recorded electronically. eMRs have several advantages over paper documents. Data can be easily tracked over time, clinicians are able to enter data at the point of patient care and data can be modified and updated quickly [3]. eMRs also have templates clinicians can use, this ensures patient details are entered comprehensively and also helps clinicians to save time [3]. PowerChart Maternity (PCM) is a single point of entry for clinicians to 
document a mother's pregnancy from their first antenatal visit through to their postpartum follow-up visit. Clinicians can at-a-glance have access to the full antenatal workflow. The Electronic Medication Management (eMeds) system ensures that the delivery of medications to patients is electronically supported and provides access to patient information and clinical decision support in real time. The implementation of eMeds helps to increase patient safety and reduce medication errors and associated adverse events. These bedside information technologies require the clinician to use a computer system with a network that will ensure the transfer and storage of health information [3].

The benefits to health services of bedside information technology such as eMRs, eMeds and PCM are well documented but we know little of the patient experience of bedside information technology practices. As health services seek to further invest in bedside technologies like eMR, eMeds and PCM, it is timely to explore patient experiences and perceptions regarding this.

This research project aims are:

1. To explore patients' experiences and perceptions regarding the use of bedside mobile technology.

2. To explore the patient experience-related impacts of introducing eMeds and PCM.

\section{Method}

The research project was conducted at The Canterbury Hospital, a metropolitan acute general hospital located in inner western Sydney.

A pre and post intervention, mixed method study using a quantitative patient experience questionnaire and qualitative semi-structured patient interviews was utilised. Three wards were involved with the study; a medical ward, a surgical ward and a maternity ward. Patients whose length of stay was forty eight hours or longer were asked to complete the questionnaire. If patients did not meet the selection criteria they were not approached. Patients that wanted to further share their experience following questionnaire completion, were asked to participate in a semi- structured interview.

The baseline data (pre-data) was collected in early 2019. The implementation of eMeds and PCM systems were introduced on participating wards in end of 2019. The post data collection was conducted in 2020 and 2021. There were designated data collection months to invite the patients to complete the questionnaires. Consecutive eligible patients were approached for involvement during these periods.

\subsection{Questionnaires}

The questions in the questionnaire were co-created by the research team, the Health Informatics team and the Nurse Consultant for Patient and Family Centred Care Research utilising the patient and family centred care model. Four domains were canvassed in both pre and post questionnaires (i) personal contact with clinical staff (four items); ii) awareness of computers and electronic devices at the bedside (four items); iii) medical records (two items); and iv) care overall (five items). Participants responded against items using a five point Likert scale (where $5=$ strongly agree and $1=$ strongly disagree). Personal demographics were also recorded. There was also the option for patients to leave comments at the end of each domain. $\mathrm{N}=82$ completed responses were obtained at 
the pre-implementation data collection point and $n=98$ were obtained for the post implementation point.

\subsection{Patient Interviews}

The interview involved eight open ended questions. Interviews were of approximately 15 minute duration.

The questions asked were:

Q1: Did you notice the presence of technology at your bedside?

Follow ups: If you did, what kinds of things were there? Were these explained to you?

Q2: Can you say how the use of bedside mobile technology affected your care?

Q3: Can you talk about the level of communication you have with clinical staff?

Follow ups: Are there things you haven't had the opportunity to communicate about, but would like to?

Q4: Do you feel that the use of bedside mobile technology has in any way depersonalised your experience/hospital stay?

Follow ups: If yes, can you describe how this affected you? If no, can you describe how you integrated the bedside technology into your experience?

Q5: How has bedside mobile technology enhanced the care you received?

Q6: How has bedside mobile technology hindered the care you received?

Q7: Can you talk about anything you've noticed regarding how bedside mobile technology has impacted on staff?

Q8: Overall, do you feel like you are receiving appropriate face to face care at the bedside?

Follow ups: What would you like to see improve regarding the face to face aspect of care?

Fifteen patient interviews were conducted during the pre and post data collection phases. Thematic analysis was used to identify the main themes arising from interview transcripts. 


\section{Results}

\subsection{Questionnaires}

Table 1 outlines participant characteristics in the two sample groups. No significant differences were noted on gender age or culturally and linguistically diverse (CALD) status.

Table 1. Sample characteristics.

\begin{tabular}{|c|c|c|c|c|}
\hline & $\begin{array}{l}\text { Pre } \\
(\mathrm{n}=82) \\
\%\end{array}$ & $\begin{array}{l}\text { Post } \\
(\mathrm{n}=98) \\
\%\end{array}$ & $\begin{array}{l}\text { Difference } \\
x^{2}\end{array}$ & $\mathrm{P}$ \\
\hline Gender & F: 67.1 & F: 54.2 & 2.8 & 0.10 \\
\hline Age range & $\begin{array}{lc}<30: & 35.8 \\
30-49: & 14.8 \\
50-69: & 34.6 \\
>69: & 14.8\end{array}$ & $\begin{array}{ll}<30: & 35.7 \\
30-49: & 18.4 \\
50-69: & 35.7 \\
>69: & 10.2\end{array}$ & 2.4 & 0.79 \\
\hline CALD & Y: 35.4 & Y: 44.9 & 1.7 & 0.19 \\
\hline
\end{tabular}

Most participants expressed satisfaction with care provided overall (median score $=$ 5 in both groups). The domain where most pre vs post change was noted lay in the 'Computers and electronic devices at the bedside' domain (Table 2). Awareness of bedside devices and satisfaction about device presence was significantly higher at posttest. Perceptions re staff frustration re working with devices were also significantly more positive at the post-test point.

Table 2. Domain: Computers and electronic devices at the bedside.

\begin{tabular}{|l|l|l|l|}
\hline Computers and electronic devices at the bedside & $\begin{array}{l}\text { Median pre } \\
(\mathrm{n}=82)\end{array}$ & $\begin{array}{l}\text { Median post } \\
(\mathrm{n}=98)\end{array}$ & $\begin{array}{l}\text { Difference (Mann- } \\
\text { Whitney U Test) }\end{array}$ \\
\hline My bedside had a lot of electronic devices & 3 & 3 & 0.41 \\
\hline $\begin{array}{l}\text { I was aware of the use of computers by clinical } \\
\text { staff, as part of my bedside care }\end{array}$ & 4 & 5 & $<0.001$ \\
\hline $\begin{array}{l}\text { I was happy with the amount of electronic devices } \\
\text { at my bedside. }\end{array}$ & 4 & 5 & 0.001 \\
\hline $\begin{array}{l}\text { The staff appeared frustrated when using } \\
\text { computers at my bedside }\end{array}$ & 1 & 1 & 0.009 \\
\hline
\end{tabular}

Significant differences were not noted for the 'personal contact with clinical staff' domain, which canvassed perception regarding acknowledgement and face to face time with staff. Similar findings were noted for the overall care quality domain, though agreement with one item in this domain 'I felt that my care was centred on me as a person' was significantly higher $(\mathrm{P}=0.02)$ at post-test. 
Two items were canvassed in the medical records domain of the questionnaire. It was noted that happiness regarding digital rather than paper storage of records slightly deteriorated at post-test $(\mathrm{P}=0.06)$, though concern about who has access to the record did not change between the two time points.

Among the three demographic characteristics which were assessed against questionnaire items cross-sectionally, age was the most prominent influencer of attitude. Concern regarding who has access to the record was more prominent among younger respondents and older age was positively associated with awareness of the use of computers at the bedside.

\subsection{Patient Interviews}

Thematic analysis of pre and post interview transcripts identified two sentinel thematic domains which related to 'efficiency' and 'expectations'. Patients identified timely or efficient access to their health information (by both them and their clinicians) as important. Marked differences in thematic content was not noted between the pre and post samples.

The following two quotes reflect a lot of the patient commentary about 'efficiency':

“Without computers I don't think everything would have gone as smoothly ... computers and technology definitely helps in the hospital",."

"I think that it actually works better for me as I can see the doctors on their rounds, they bring it with them and they are typing, but they they're including me and talking about my hip".

The second prominent theme related to expectations. Patients commonly expressed a view that technology is widely used in society and that health should be no exception.

Examples of patient comments about expectations:

"I think that is what I expect to see, computers in hospitals".

"I am used to computers, phones, whatever at home and at work".

\section{Discussion}

The "awareness of computers and electronic devices at the bedside" and "medical records" domains of the questionnaire specifically targeted technology-related patient perception and attitude. Pre vs post comparative results indicate that awareness of and happiness with the amount of devices at the bedside increased at post-test. Perceptions regarding staff frustration were more positive at post-test, though this was not perceived to be a major problem at either time point (the median score in both samples for this item was 1). Perceptions regarding care quality and personal contact with staff were similar at both time points. These findings suggest that patients noticed the change in amount of bedside technology and that they were satisfactory with this. The positivity of the patient experience was found to be unaffected. Most questionnaire participants at both time points were untroubled by the eMR concept. Interestingly, older age was found to be the most important associate of positive perception about access to digital records. This 
finding may be counter-intuitive to some, who might expect that advancing age could be associated with distrust in relation to technology.

The findings from the qualitative study emphasised efficiency and expectation as key factors around which patients locate bedside technology within their larger experience of hospitalisation. The notion that people expect to find technology in all areas of their lives (including their hospital bedside) is interesting, and suggestive that contemporary societal practices are likely to help people adjust to digital healthcare, over time.

\section{Conclusion}

As the digital transformation of healthcare continues and accelerates, it is important that healthcare organisations retain the primacy of the patient and family-centred care ethos. This research project yields encouraging findings for clinical nurses and nurse informaticists who may be concerned about negative impacts on patient care associated with increasing bedside digitisation. Such a hypothesis is not supported by the findings of this study.

\section{Acknowledgements}

We would like to acknowledge the generous support of the executive, staff and patients of The Canterbury Hospital and Sydney Local Health District. We would also like to acknowledge staff that have assisted with the data collection, Leanne Wiseman and Eloise Clarke.

\section{References}

[1] Aiken L, Sermeus W, Van den Heede K. (2012) Patient safety, satisfaction, and quality of hospital care: cross sectional surveys of nurses and patients in 12 countries in Europe and the United States. BMJ 2012; 344 .

[2] Doyle C, Lennox L, Bell D. (2012), A systematic review of evidence on the links between patient experience and clinical safety and effectiveness. BMJ.

[3] Okpala PC. The Electronic Medical Record (EMR). Journal of Applied Medical Sciences, vol. 2, no. 2, (2013), 79-85.

[4] Westbrook J, Li L, Georgiou A, Paoloni R, Cullen J. Impact of an electronic medication management system on hospital doctors' and nurses' work: a controlled pre-post, time and motion study. J Am Med Inform Assoc 2013;20:1150-1158. 